\title{
Fermion Tunneling from Dynamical Horizons
}

\author{
R. Di Criscienzo* \\ Department of Physics-University of Toronto, \\ 60 St. George Street, Toronto, Ontario, M5S 1A7, Canada \\ L. $\operatorname{Vanzo}^{\dagger}$ \\ Dipartimento di Fisica, Università di Trento \\ and INFN, Gruppo Collegato di Trento \\ Via Sommarive 14, 38100 Povo (TN), Italia
}

\begin{abstract}
The instability against emission of fermionic particles by the trapping horizon of an evolving black hole is analyzed using the Hamilton-Jacobi tunneling method. This method automatically selects one special expression for the surface gravity of a changing horizon. The results also apply to point masses embedded in an expanding universe. As a bonus of the tunneling method, we gain the insight that the surface gravity still defines a temperature parameter as long as the evolution is sufficiently slow that the black hole pass through a sequence of quasi-equilibrium states, and that black holes should be semi-classically unstable even in a hypothetical world without bosonic fields.
\end{abstract}

PACS numbers: $\quad 04.70 .-5,04.70 . B w, 04.70 . D y$

\section{Introduction}

In a previous paper [1] we considered the quantum instability of dynamical black holes using a variant of the tunneling method introduced by Parikh and Wilczek [2], according to which the Hawking effect can be considered as a kind of tunneling transition through the horizon of the black hole (BH). Probably, for such matters it was first applied in condensed matter physics, specifically to exotic phenomena involving the analogue of horizons in superfluid ${ }^{3} \mathrm{He}$ films [3]. The method was refined and extended to more general cases in [4] and others papers as well [5], including the back reaction effects [6] and its extension to non-commutative Schwarzschild space-time [7]. For criticism and counter criticism see also [8], and [9] for the relation between tunneling and thermodynamics.

The Hawking's effect should not be confused with another tunneling process, the escape of the particle to infinity through the potential barrier surrounding the horizon, and about which the Parikh-Wilczek method has nothing to say.

The variant mentioned above is the Hamilton-Jacobi method introduced in [10,11], so called after the appearance of a complete comparison analysis with the Parikh-Wilczek method done in [12]. The tunneling method provides not only new physical insight to an understanding of the black hole radiation, but is also a powerful way to compute the surface gravity for a vast range of solutions. Now for non stationary black holes, termed dynamical black holes in [1], things are not so simple and even the possibility of Hawking radiation is in principle questionable, since in general a changing horizon is not a null hypersurface, although it is still one of infinite red shift. In particular, several definitions of the surface gravity for evolving horizons have been proposed in the past, all fitting the first law of $\mathrm{BH}$ mechanics more or less

*rdicris@physics.utoronto.ca

†vanzo@science.unitn.it 
equally well. A comparison is discussed throughly in [13]. Among these the one proposed implicitly by Visser [10] and more explicitly by Hayward and Kodama [14,15] is the most interesting to us, as it is the one the tunneling method leads to. Still different, otherwise reasonable, resuls are advocated for expanding cosmological black holes $[16,17]$, but we shall not dwell about them here.

As we said above, a way to understand Hawking radiation is by means of tunneling of particles through black-hole horizons. Such tunneling approach uses the fact that the WKB approximation of the tunneling probability for the classical forbidden trajectory from inside to outside the horizon is:

$$
\Gamma \propto e^{-\frac{2}{\hbar} \operatorname{Im} I}
$$

where $I$ is the classical action of the trajectory, to leading order in $\hbar$. What kind of particles do we expect to find in the Hawking radiation spectrum of a black-hole? In principle, all the Standard Model particles. However, most of the calculations in literature have been performed just for scalar fields, except in [18], where a detailed study of spin one-half emission was considered for stationary black holes, and [19] for the special case of the BTZ black hole. What we are going to check is that the tunneling approach via the Hamilton-Jacobi method is consistent with the Kodama-Hayward prediction even for 1/2-spin particles in dynamical black holes and that, as a consequence, they should be semi-classically unstable even in a world with no bosonic fields.

\section{Bardeen-Vaidya space-times and cosmological black holes}

Let us consider the Bardeen - Vaidya metric (BV) in $D=4$ dimensions $[20,21]$

$$
d s^{2}=-e^{2 \psi(r, v)} A(r, v) d v^{2}+2 e^{\psi(r, v)} d v d r+r^{2} d \omega^{2},
$$

where $v$ is an advanced time null coordinate, $d \omega^{2}=d \theta^{2}+\sin ^{2} \theta d \phi^{2}$ and, in the simplest case we have in mind, $A(r, v)$ is just

$$
A(r, v)=1-\frac{2 m(r, v)}{r}
$$

The inverse metric is given by:

$$
g^{\mu \nu}=\left(\begin{array}{cccc}
0 & e^{-\psi(r, v)} & 0 & 0 \\
e^{-\psi(r, v)} & A(r, v) & 0 & 0 \\
0 & 0 & \frac{1}{r^{2}} & 0 \\
0 & 0 & 0 & \frac{1}{r^{2} \sin ^{2} \theta}
\end{array}\right) .
$$

This class of metrics possesses - under very general conditions - a trapping horizon (TH), as defined by Hayward [14], which in the present case is given by the equation $A(r, v)=0$. This defines a curve $r=r_{H}(v)$ giving the location of the apparent horizon; the quantity $E=m\left(r_{H}(v), v\right)$ is the Misner-Sharp mass [22] of the horizon, in term of which the horizon will be trapping if $m^{\prime}\left(r_{H}, v\right)<1 / 2$, a prime denoting the radial derivative. The TH proved to be the key concept in such matters as particles emission and gravitational entropy (for a very recent discussion see [23]), and can be applied successfully even to higher dimensional Vaidya space-times [24].

According to the Kodama-Hayward theory, to such TH is associated a geometrical surface gravity

$$
\kappa(v)=\left.\frac{A^{\prime}(r, v)}{2}\right|_{T H}=\frac{1}{2 r_{H}}-\frac{m^{\prime}\left(r_{H}, v\right)}{r_{H}} .
$$

We see the meaning of the trapping condition: it ensures the positivity of the surface gravity. The second example we are interested in is the McVittie solution [25] for a point mass in 
a Friedmann-Robertson-Walker flat cosmology. In isotropic spatial coordinates it is given by $[25]$

$$
d s^{2}=-A(\rho, t) d t^{2}+B(\rho, t)\left(d \rho^{2}+\rho^{2} d \omega^{2}\right)
$$

with

$$
A(\rho, t)=\left[\frac{1-\frac{m}{a(t) \rho}}{1+\frac{m}{a(t) \rho}}\right]^{2}, \quad B(\rho, t)=a(t)^{2}\left[1-\frac{m}{a(t) \rho}\right]^{2}
$$

When the mass parameter $m=0$, it reduces to a spatially flat FRW solution with scale factor $a(t)$; when $a(t)=1$ it reduces to the Schwarzschild metric with mass $m$. In four dimensions this solution has had a strong impact on the general problem of matching the Schwarzschild solution with cosmology, a problem faced also by Einstein and Dirac. Besides McVittie, it has been extensively studied by Nolan in a series of papers [26]. To put the metric in the general form of Kodama theory, we use what may be called the Nolan gauge, in which the metric reads

$$
d s^{2}=-\left(A_{s}-H^{2}(t) r^{2}\right) d t^{2}+A_{s}^{-1} d r^{2}-2 A_{s}^{-1 / 2} H(t) r d r d t+r^{2} d \omega^{2}
$$

where $H(t)=\dot{a} / a$ is the Hubble parameter and, for example, in the charged case, $A_{s}=$ $1-2 m / r+q^{2} / r^{2}$. In passing to the Nolan gauge a choice of sign in the cross term $d r d t$ has been done, corresponding to an expanding universe; the transformation $H(t) \rightarrow-H(t)$ changes this into a contracting one. In the following we shall consider $q=0$; then the Einstein-Friedmann equations read

$$
3 H^{2}=8 \pi \rho, \quad 2 A_{s}^{-1 / 2} \dot{H}(t)+3 H^{2}=-8 \pi p .
$$

It follows that $A_{s}=0$, or $r=2 m$, is a curvature singularity similar to $r=0$ in FRW models, namely it is a big bang singularity. When $H=0$ one has the Schwarzschild solution. The term $H^{2} r^{2}$ in the metric strongly resembles a varying cosmological constant; in fact if $H$ is constant the metric reduces to the Schwarzschild-de Sitter solution in Painlevé coordinates. The trapping horizon is the root of $A_{s}=H^{2} r_{H}^{2}$, and is time dependent. The Misner-Sharp mass and the geometrical surface gravity are, respectively,

$$
\begin{aligned}
& E=m+\frac{1}{2} H(t)^{2} r_{H}^{3}, \\
& \kappa(t)=\frac{m}{r_{H}^{2}}-H^{2} r_{H}-\frac{\dot{H}}{2 H} .
\end{aligned}
$$

Note that $E=r_{H} / 2$. In the static cases everything agrees with the standard results. The surface gravity has an interesting expression in terms of the sources of Einstein equations and the Misner-Sharp mass. Let $T_{2 D}$ be the reduced trace of the stress tensor in the space normal to the sphere of symmetry, evaluated on the horizon. For the Vaidya-Bardeen metric it is, by Einstein's equations,

$$
T_{2 D}=T_{v}^{v}+T_{r}^{r}=-\frac{1}{2 \pi r_{H}} \frac{\partial m}{\partial r}{ }_{\mid r=r_{H}}
$$

For the McVittie's solution, this time by Fredmann's equations (2.7) one has

$$
T_{2 D}=-\rho+p=-\frac{1}{4 \pi}\left(3 H^{2}+\frac{\dot{H}}{H r_{H}}\right)
$$

We have then

$$
\kappa=\frac{E}{r_{H}^{2}}+2 \pi r_{H} T_{2 D}
$$

It is worth mentioning the pure FRW case, i.e. $A_{s}=1$, for which $\kappa(t)=-H(t)-\dot{H} / 2 H$. We feel that these expressions for the surface gravity are non trivial and display deep connections with the emission process. We now proceed to analyze the Dirac equation in BV space-time. Since the Hawking's effect is a phase phenomenon, which is why the tunneling method works so well, we expect the spinor amplitudes to play a minor role. We verify this for the BV "shining star", but use a more straightforward method for the McVittie's solution. 


\section{Dirac equation for BV metric}

The Dirac equation is,

$$
\left(\gamma^{\mu} D_{\mu}+\frac{m}{\hbar}\right) \Psi(v, r, \theta, \phi)=0,
$$

where

$$
\begin{aligned}
D_{\mu} & =\partial_{\mu}+\frac{i}{2} \Gamma_{\mu}^{\alpha}{ }^{\beta} \Sigma_{\alpha \beta}, \\
\Sigma_{\alpha \beta} & =\frac{i}{4}\left[\gamma_{\alpha}, \gamma_{\beta}\right]_{-} .
\end{aligned}
$$

The $\gamma^{\mu}$ - matrices satisfy the Clifford algebra,

$$
\left[\gamma_{\alpha}, \gamma_{\beta}\right]_{+}=2 g_{\alpha \beta} \mathbb{I},
$$

where $\mathbb{I}$ is the $(4 \times 4)$-identity matrix. In order to get the Dirac- $\gamma$ matrices for the BV metric at hand, we define firstly a tetrad of orthogonal vectors $E_{\mu}^{a}$ s.t.

$$
\eta_{a b} E_{\mu}^{a} E_{\nu}^{b}=g_{\mu \nu} .
$$

The convention is that (first) latin indices are Minkovskian so they run over $(0,1,2,3)$; greek indices are coordinate indices so they run over $(v, r, \theta, \phi)$.

Of course there are many different tetrads, but the simplest choice is the following:

$$
\begin{aligned}
E_{v}^{a} & =\left(e^{\psi} \sqrt{A}, 0,0,0\right), \\
E_{r}^{a} & =\left(-\frac{1}{\sqrt{A}}, \frac{1}{\sqrt{A}}, 0,0\right), \\
E_{\theta}^{a} & =(0,0, r, 0), \\
E_{\phi}^{a} & =(0,0,0, r \sin \theta) .
\end{aligned}
$$

The $\gamma_{\mu}$-matrices are expressed in terms of the tetrad in the following way:

$$
\gamma_{\mu}=\gamma_{a} E_{\mu}^{a} .
$$

With our choice (3.6) - (3.9), it turns out that

$$
\begin{aligned}
\gamma_{v} & =e^{\psi} \sqrt{A} \gamma_{0}, \\
\gamma_{r} & =\frac{1}{\sqrt{A}}\left(\gamma_{1}-\gamma_{0}\right), \\
\gamma_{\theta} & =r \gamma_{2}, \\
\gamma_{\phi} & =r \sin \theta \gamma_{3} ;
\end{aligned}
$$

and

$$
\begin{aligned}
\gamma^{v} & =\frac{e^{-\psi}}{\sqrt{A}}\left(\gamma_{1}-\gamma_{0}\right), \\
\gamma^{r} & =\sqrt{A} \gamma_{1}, \\
\gamma^{\theta} & =\frac{1}{r} \gamma_{2}, \\
\gamma^{\phi} & =\frac{1}{r \sin \theta} \gamma_{3} .
\end{aligned}
$$

We can also express the

$$
\gamma^{5} \stackrel{\text { def }}{=} i \gamma^{v} \gamma^{r} \gamma^{\theta} \gamma^{\phi}=\frac{i e^{-\psi}}{r^{2} \sin \theta}\left(\mathbb{I}-\gamma_{0} \gamma_{1}\right) \gamma_{2} \gamma_{3} .
$$


All what we need now is a convenient representation of the Dirac- $\gamma_{a}$ matrices satisfying $\left[\gamma_{a}, \gamma_{b}\right]_{+}=2 \eta_{a b}$ with $\eta=\operatorname{diag}(-1,1,1,1)$.

$$
\begin{aligned}
\gamma_{0} & =\left(\begin{array}{cc}
i & 0 \\
0 & -i
\end{array}\right) ; \\
\gamma_{1} & =\left(\begin{array}{cc}
0 & \sigma_{3} \\
\sigma_{3} & 0
\end{array}\right) ; \\
\gamma_{2} & =\left(\begin{array}{cc}
0 & \sigma_{1} \\
\sigma_{1} & 0
\end{array}\right) ; \\
\gamma_{3} & =\left(\begin{array}{cc}
0 & \sigma_{2} \\
\sigma_{2} & 0
\end{array}\right) .
\end{aligned}
$$

The $\sigma$-matrices are the Pauli matrices satisfying the usual relations:

$$
\sigma_{i} \sigma_{j}=\mathbb{I}^{(2 \times 2)} \delta_{i j}+i \varepsilon_{i j k} \sigma_{k}, \quad i, j, k=1,2,3 .
$$

By virtue of (2.1) and (2.3), one calculates the $\Gamma_{\mu}^{\alpha}{ }^{\beta}$ symbols required in (3.2). We list the result in the footnote below 1 for the sake of completness. Next, we should calculate the $\Sigma_{\alpha \beta}$ defined in (3.3): a task that Maple can do very quickly; in the end, it remains to evaluate the combination of such results, namely

$$
\not \partial \Psi+i\left[\frac{1}{2} \gamma^{\mu} \Gamma_{\mu}^{\alpha}{ }^{\beta} \Sigma_{\alpha \beta} \Psi\right]+\frac{m}{\hbar} \Psi=0 .
$$

Let us employ the following ansatz for the spin-up Dirac field2:

$$
\Psi(v, r, \theta, \phi)_{\uparrow}=\left(\begin{array}{c}
\Xi(v, r, \theta, \phi) \\
0 \\
\Omega(v, r, \theta, \phi) \\
0
\end{array}\right) \exp \left[\frac{i}{\hbar} I_{\uparrow}(v, r, \theta, \phi)\right] .
$$

Plugging the ansatz (3.26) into Dirac equation (3.25), it turns out that the term in square brackets is of order $O(\hbar)$. Thus, we do not need to work out its precise form, since in the $\hbar \rightarrow 0$ limit it vanishes. To leading order in $\hbar$ equation (3.25) becomes $0=(\hbar \not \partial+m) \Psi_{\uparrow}+O(\hbar)$, or

$$
\begin{aligned}
0= & \exp \left[\frac{i}{\hbar} I_{\uparrow}(v, r, \theta, \phi)\right] \times\left\{\frac{e^{-\psi}}{\sqrt{A}}\left(\begin{array}{c}
i \Xi \partial_{v} I_{\uparrow}-\Omega \partial_{v} I_{\uparrow} \\
0 \\
-\Xi \partial_{v} I_{\uparrow}-i \Omega \partial_{v} I_{\uparrow} \\
0
\end{array}\right)\right. \\
& +\sqrt{A}\left(\begin{array}{c}
-\Omega \partial_{r} I_{\uparrow} \\
0 \\
-\Xi \partial_{r} I_{\uparrow} \\
0
\end{array}\right)+\frac{1}{r}\left(\begin{array}{c}
0 \\
-\Omega \partial_{\theta} I_{\uparrow} \\
0 \\
-\Xi \partial_{\theta} I_{\uparrow}
\end{array}\right)+\frac{1}{r \sin \theta}\left(\begin{array}{c}
0 \\
-i \Omega \partial_{\phi} I_{\uparrow} \\
0 \\
-i \Xi \partial_{\phi} I_{\uparrow}
\end{array}\right)+i m\left(\begin{array}{c}
\Xi \\
0 \\
\Omega \\
0
\end{array}\right) .
\end{aligned}
$$

${ }^{1}$ The non-vanishing $\Gamma_{\mu}^{\alpha}{ }^{\beta}=g^{\beta \nu} \Gamma_{\mu \nu}^{\alpha}$ are:

$$
\begin{aligned}
& \Gamma_{v}^{v} v^{v}=e^{-\psi} \dot{\psi}+\frac{A^{\prime}}{2}+A \psi^{\prime}, \quad \Gamma_{\theta}^{v}{ }^{\theta}=-\frac{e^{-\psi}}{r}, \quad \Gamma_{\phi}^{v}{ }^{\phi}=-\frac{e^{-\psi}}{r} ; \\
& \Gamma_{v}^{r} v^{v}=-\frac{A^{\prime}}{2}-A \psi^{\prime}, \quad \Gamma_{v}^{r}{ }^{r}=-\frac{\dot{A}}{2}, \quad \Gamma_{r}^{r}{ }^{r}=-\frac{A^{\prime}}{2}, \Gamma_{r}^{r}{ }^{v}=e^{-\psi} \psi^{\prime}, \quad \Gamma_{\theta}^{r} \theta=-\frac{A}{r}, \quad \Gamma_{\phi}^{r}{ }^{\phi}=-\frac{A}{r} ; \\
& \Gamma_{r}^{\theta}{ }^{\theta}=\frac{1}{r^{3}}, \quad \Gamma_{\phi}^{\theta}{ }^{\phi}=-\frac{\cos \theta}{r^{2} \sin \theta}, \quad \Gamma_{\theta}^{\phi}{ }^{\phi}=\frac{\cos \theta}{r^{2} \sin ^{3} \theta} ; \\
& \Gamma_{\phi}^{\phi}{ }^{\theta}=\frac{\cos \theta}{r^{2} \sin \theta}, \quad \Gamma_{r}^{\phi}{ }^{\phi}=\frac{1}{r^{3} \sin ^{2} \theta} \text {. }
\end{aligned}
$$

${ }^{2}$ We shall perform a detailed analysis only for the spin-up case, being confident that mutatis mutandis everything applies in the same way to the spin-down case. 
Thus, we get the following equations:

$$
\begin{aligned}
v: & \frac{i \Xi}{\sqrt{A}}\left(e^{-\psi} \partial_{v}\right) I_{\uparrow}-\frac{\Omega}{\sqrt{A}}\left(e^{-\psi} \partial_{v}\right) I_{\uparrow}-\sqrt{A} \Omega \partial_{r} I_{\uparrow}+i \Xi m=0 ; \\
r: & -\frac{\Omega}{r}\left(\partial_{\theta} I_{\uparrow}+\frac{i}{\sin \theta} \partial_{\phi} I_{\uparrow}\right)=0 ; \\
\theta: & -\frac{\Xi}{\sqrt{A}}\left(e^{-\psi} \partial_{v}\right) I_{\uparrow}-\frac{i \Omega}{\sqrt{A}}\left(e^{-\psi} \partial_{v}\right) I_{\uparrow}-\sqrt{A} \Xi \partial_{r} I_{\uparrow}+i \Omega m=0 ; \\
\phi: & -\frac{\Xi}{r}\left(\partial_{\theta} I_{\uparrow}+\frac{i}{\sin \theta} \partial_{\phi} I_{\uparrow}\right)=0,
\end{aligned}
$$

and the Kodama vector $K=e^{-\psi} \partial_{v}$ has been put in evidence throughout. $K$ plays the role of the Killing vector $\partial_{t}$ for dynamical black-holes. Therefore, it makes sense the following ansatz for the action:

$$
I_{\uparrow}=-\int d v e^{\psi(r, v)} E+W(r)+J(\theta, \phi),
$$

which inserted into (3.28) - (3.31) gives:

$$
\begin{array}{ll}
v: & \frac{1}{\sqrt{A}}(i \Xi-\Omega) E-\sqrt{A} \Omega W^{\prime}(r)+i \Xi m=0 ; \\
r: & -\frac{\Omega}{r}\left(J_{\theta}(\theta, \phi)+\frac{i}{\sin \theta} J_{\phi}(\theta, \phi)\right)=0 ; \\
\theta: & -\frac{1}{\sqrt{A}}(\Xi+i \Omega) E-\sqrt{A} \Xi W^{\prime}(r)+i \Omega m=0 ; \\
\phi \quad: \quad & -\frac{\Xi}{r}\left(J_{\theta}(\theta, \phi)+\frac{i}{\sin \theta} J_{\phi}(\theta, \phi)\right)=0 .
\end{array}
$$

(3.34) and (3.36) imply that $J(\theta, \phi)$ is a complex function. The same solution for $J$ is obtained for the spin-down case, then its contribution to the rate emission $\Gamma$ cancels out and we can forget about it. As regard the remaining equations, we have

1. $\Xi=i \Omega$, then

$$
W^{\prime}(r)=-\frac{2 E}{A(r, v)}
$$

2. $\Xi=-i \Omega$, then

$$
W^{\prime}(r)=0 \text {, }
$$

both in the massless and massive cases. Solution (3.38) has not to be considered surprising, since

$$
\gamma^{5} \Psi=\left(\begin{array}{c}
i \Xi-\Omega \\
0 \\
\Xi+i \Omega \\
0
\end{array}\right) .
$$

meaning that case 2. corresponds to the incoming particle absorbed in the classical limit with probability $\mathscr{P}[$ incoming $]=1$. The emission process is described instead by case 1 . As explained elsewhere [1] this implies that

$$
\operatorname{Im} W(r)=-\operatorname{Im} \int d r \frac{E}{A(r, v) / 2}=\frac{2 \pi i E}{A^{\prime}\left(r_{H}(v), v\right)} .
$$

In the end we get,

$$
\Gamma \frac{1.1}{\propto} e^{-\frac{2 \pi}{A^{\prime}\left(r_{H}(v), v\right) / 2} E}, \quad \Longrightarrow \quad T=\frac{A^{\prime}(r, v)_{\left.\right|_{r=r_{H}}(v)}}{4 \pi},
$$

confirming the predictions of the Kodama-Hayward theory (2.4). We conclude that our dynamical black hole is unstable against the emission of spinor particles. It is essential, for this result to hold, that the black hole be slowly evolving on the timescale of the wave, since otherwise no meaningful notion of a frequency is available. 


\section{MacVittie cosmological black hole}

The preceding calculations showed that the derivatives of the action were strongly mixed by the matrix structure of the Dirac equation. Nevertheless things combined so that only the radial derivative of the action was really important. Hence we will not study the full Dirac equation in the following, but use instead a shortcut. Writing as before

$$
\Psi=U \exp \left[\frac{i}{\hbar} I(t, r, \theta, \phi)\right] .
$$

where $U$ is a slowly varying spinor amplitude, from the Dirac equation we get

$$
\not D U+\hbar^{-1}(i \not \partial I+m) U=0
$$

In the semi-classical limit the second term dominates, so $(i \not \partial I+m) U=0$; thus the matrix $(i \not \supset I+m)$ must be singular, or equivalently,

$$
g^{\mu \nu} \partial_{\mu} I \partial_{\nu} I+m^{2}=0
$$

The action will have a simple pole at the location of the trapping horizon affecting to outgoing modes, so for these we also neglect the mass term. Then for an outgoing particle we find

$$
\partial_{r} I=-F(r, t)^{-1} \partial_{t} I,
$$

where

$$
F(r, t)=\sqrt{A_{s}(r)}\left(\sqrt{A_{s}(r)}-r H(t)\right) .
$$

We pick the imaginary part by expanding this function at the horizon along a future null direction, using the fact that for two neighbouring events on a null direction in the metric (2.6), one has $t-t_{0}=\left(2 H_{0}^{2} r_{0}^{2}\right)^{-1}\left(r-r_{0}\right)$, where $H_{0}=H\left(t_{0}\right)$. We find the result

$$
F(r, t)=\left(\frac{1}{2} A_{s}^{\prime}\left(r_{0}\right)-r_{0} H_{0}^{2}-\frac{\dot{H}_{0}}{2 H_{0}}\right)\left(r-r_{0}\right)=\kappa_{0}\left(r-r_{0}\right)
$$

where this time $r_{0}=r_{H}\left(t_{0}\right)$. From this equation we see that $\partial_{r} I$ has a simple pole at the trapping horizon; hence, making use again of Feynman $i \epsilon$-prescription, one finds

$$
\operatorname{Im} I=\pi \kappa\left(t_{0}\right)^{-1} \omega\left(t_{0}\right),
$$

where $\omega(t)=\partial_{t} I$ is the energy at time $t$, in complete agreement with the geometric evaluation of the previous sections. However, we stress that a full justification of the given shortcut really requires the full spinor amplitudes, as we showed above while discussing the BV black hole.

\section{References}

[1] R. Di Criscienzo, M. Nadalini, L. Vanzo, S. Zerbini and G. Zoccatelli, Phys. Lett. B 657 (2007) 107.

[2] M. K. Parikh and F. Wilczek, Phys. Rev. Lett. 85, 5042 (2000).

[3] G. E. Volovik, Pisma Zh. Eksp. Teor. Fiz. 69 (1999) 662 [JETP Lett. 69 (1999) 705]; A. Calogeracos and G.E. Volovik, JETP Lett. 69, 281 (1999); G. E. Volovik, arXiv:cond-mat/9902171.

[4] S. Shankaranarayanan, T. Padmanabhan and K. Srinivasan, Class. Quant. Grav. 19, 2671 (2002); E. C. Vagenas, Phys. Lett. B 559, 65 (2003); A. J. M. Medved, Phys. Rev. D66, 124009 (2002); T. Padmanabhan, Mod. Phys. Lett. A 19, 2637 (2004).

[5] K. Srinivasan and T. Padmanabhan, Phys. Rev. D 60, 24007 (1999); S. Hemming and E. Keski-Vakkuri, Phys. Rev. D 64, 044006 (2001); Q. Q. Jiang, H. L. Li, S. Z. Yang and D. Y. Chen, Mod. Phys. Lett. A 22, 891 (2007). 
[6] A. J. M. Medved and E. C. Vagenas, Mod. Phys. Lett. A 20, 2449 (2005); M. Arzano, A. J. M. Medved and E. C. Vagenas, JHEP 0509, 037 (2005); R. Banerjee and B. R. Majhi, arXiv:0801.0200 [hep-th].

[7] R. Banerjee, B. R. Majhi and S. Samanta, arXiv:0801.3583 [hep-th].

[8] E. T. Akhmedov, V. Akhmedova and D. Singleton, Phys. Lett. B 642, 124 (2006); T. K. Nakamura, arXiv: 0706.2916 [hep-th]; P. Mitra, Phys.Lett. B648 240 (2007).

[9] T. Pilling, arXiv:0709.1624 [gr-qc].

[10] M. Visser, Int. J. Mod. Phys. D 12 (2003) 649; A. B. Nielsen and M. Visser, Class. Quant. Grav. 23 (2006) 4637.

[11] M. Angheben, M. Nadalini, L. Vanzo and S. Zerbini, JHEP 0505, 014 (2005); M. Nadalini, L. Vanzo and S. Zerbini, J. Physics A Math. Gen. 39, 6601 (2006).

[12] R. Kerner and R. B. Mann, Phys. Rev. D 76, 104010 (2006).

[13] A. B. Nielsen and J. H. Yoon, arXiv:0711.1445 [gr-qc].

[14] S. A. Hayward, Class. Quant. Grav. 15, 3147 (1998).

[15] H. Kodama, Prog. Theor. Phys. 63, 1217 (1980).

[16] V. Faraoni, Phys. Rev. D 76 (2007) 104042.

[17] H. Saida, T. Harada and H. Maeda, Class. Quant. Grav. 24 (2007) 4711.

[18] R. Kerner and R. B. Mann, arXiv:0710.0612 [hep-th].

[19] R. Li and J. R. Ren, arXiv:0802.3954 [gr-qc].

[20] P. C. Vaidya, Proc. Indian Acad. Sci. A33, 264 (1951); P. C. Vaidya, Nature 171, 260 (1953); V.V. Narlikar and P.C. Vaidya, Nature 159, 642 (1947).

[21] J.M. Bardeen, Phys. Rev. Letters 46, 382 (1981).

[22] C. W. Misner and D. H. Sharp, Phys. Rev. 136, B571 (1964);

[23] A. B. Nielsen arXiv:0802.3422 [gr-qc].

[24] J. R. Ren and R. Li, arXiv:0705.4339 [gr-qc].

[25] G. C. McVittie, Mon. Not. R. Astronomic Soc. 93, 325 (1933).

[26] B. C. Nolan, Phys. Rev. D 58, 064006 (1998); Class. Quant. Grav. 16, 1227 (1999); Class. Quant. Grav. 16, 3183 (1999). 